\title{
On the orientational distribution functions in de Vries-type smectic liquid crystals
}

\author{
Per Rudquist ${ }^{\mathrm{a}}$, Mikhail A. Osipov ${ }^{\mathrm{b}}$ and Frank Giesselmann ${ }^{\mathrm{c}}$ \\ ${ }^{a}$ Department of Microtechnology and Nanoscience, Chalmers University of Technology, Gothenburg, \\ Sweden; ${ }^{b}$ Department of Mathematics, University of Strathclyde, Glasgow, UK; ${ }^{\mathrm{c}}$ Institute of Physical \\ Chemistry, University of Stuttgart, Stuttgart, Germany
}

\begin{abstract}
ARTICLE HISTORY
Compiled May 22, 2018

ABSTRACT

In de Vries smectic liquid crystals the transition from the orthogonal smectic A (SmA) to the tilted smectic C (SmC) phase occurs with essentially no decrease in smectic layer thickness. This unusual behavior is commonly explained assuming a 'hollow cone' or 'volcano-like' orientational distribution function (ODF) of rod-like molecules in SmA and the transition to SmC being a pure disorder-order transition in the molecular tilt directions. However, even after 20 years of extensive investigations, the experimental confirmation of this ad-hoc model is still inconclusive. While optical and electrooptic studies of many de Vries smectics can be readily explained with the hollow cone model, X-ray diffraction studies as well as many polarized Raman spectroscopy studies support a broad Maier-Saupe distribution of a sugarloaf-like shape. We review and summarize X-ray, electro-optic and polarized Raman spectroscopy results on the orientational distribution functions in the SmA phases of materials claimed to be of the de Vries type and discuss how seemingly contradicting findings can both be true at the same time. Optical properties are governed by the order of the aromatic core, whereas X-ray diffraction essentially probes the order of principal molecular axes. In molecules where the core is far from collinear with the long molecular axis, the cores may exhibit a volcano ODF while the molecular axes exhibit a sugarloaf ODF.
\end{abstract}

\section{KEYWORDS}

Smectic liquid crystals; de Vries smectics; orientational distribution functions; X-ray diffraction; polarized Raman spectroscopy

\section{Introduction}

With the concept of a so-called de Vries-type smectic liquid crystal we understand a material that essentially does not change its smectic layer spacing in connection with the transition from the orthogonal smectic A ( $\mathrm{SmA})$ to the tilted smectic $\mathrm{C}(\mathrm{SmC})$ phase (Fig. 1). The first person to contemplate this possibility was Adriaan de Vries in 1977 [1]. According to his original ideas, the rod-like molecules must already be tilted in this kind of smectic A phase with a large and constant magnitude but random directions of tilt. Despite the individual molecular tilt the smectic A phase remains uniaxial and the transition to the biaxial smectic $\mathrm{C}$ state is seen as a global ordering in the molecular tilt directions. In this disorder-order transition the appearance of director tilt is coupled to an increase in orientational order and thus in optical birefringence (see Fig. 2) [2].

The model thus ad hoc predicts that there is zero layer shrinkage at the $\mathrm{A}-\mathrm{C}$ transition. This model is often referred to as the 'hollow cone' model since molecules having a fixed tilt angle $\beta$ with respect to the smectic layer normal $\mathbf{k}$ are distributed on the surface of a (molecular tilt) cone with opening angle $2 \beta$. In the more general and less extreme case the orientational 


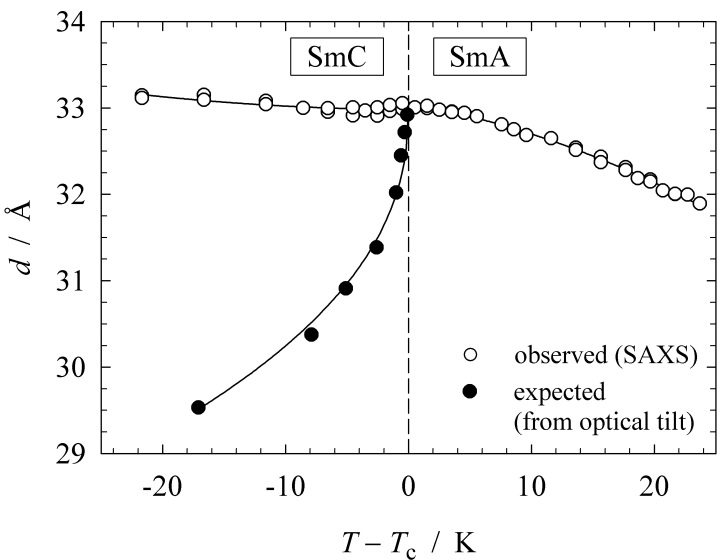

Figure 1. Temperature-dependent smectic layer spacing $d$ in the SmA and SmC phases of a de Vries-type liquid crystal (3M 8422, see Ref. [2]). The actual $d$ values measured by small-angle X-ray scattering (SAXS) are compared with the hypothetical values which are expected from the optical tilt angle in the SmC phase. From Ref. [2].
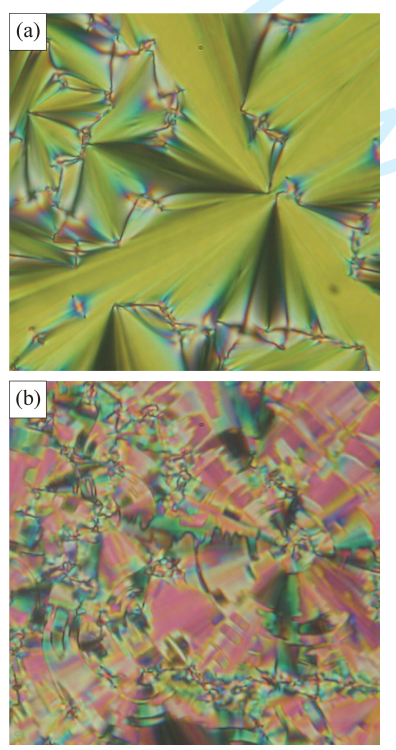

Figure 2. Texture micrographs of a 10- $\mu \mathrm{m}$ planar sample of 3M 8422 (see Ref. [2]) observed between crossed polarizers in the $\mathrm{SmA}$ phase at $71^{\circ} \mathrm{C}$ (a) and in the $\mathrm{SmC}$ phase at $47^{\circ} \mathrm{C}$ (b). The distinct change in interference color from yellowish green to red is related to the birefringence increasing in the de Vries-type tilting transition from $\Delta n=0.07$ in $\mathrm{SmA}$ to $\Delta n=0.09$ in SmC. From Ref. [2]. 
Figure 3. (a) Hollow cone model (right) leading to a volcano-like ODF in SmA (middle) and a pure disorder-order transition to SmC (left). (b) A broad sugarloaf ODF would give the same layer spacing $d_{A}$ in SmA. In both, (a) and (b), the transition to SmC (left) involves a significant increase in orientational order, which in (b) counteracts the layer shrinkage from director tilt.
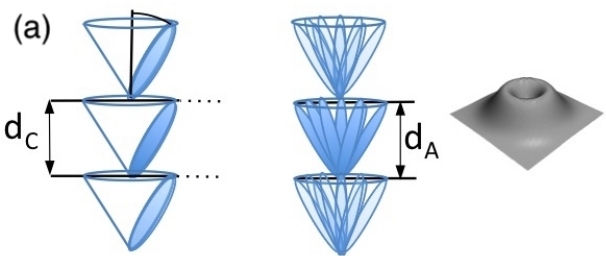

(b)
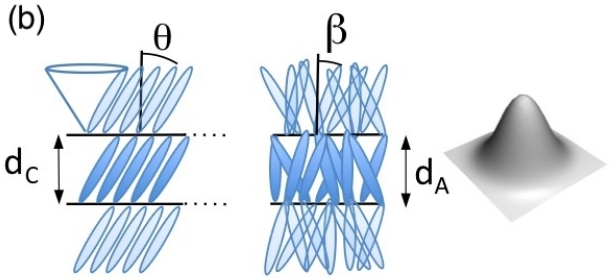

tribution function (ODF) has a volcano-like shape with a local minimum along the cone axis k (Fig. 3a). This contrasts the conventional picture of a Maier-Saupe ODF with a global maximum along $\mathbf{k}$ and a sugarloaf-like shape (Fig. $3 \mathrm{~b}$ ).

Later on, the early ideas of de Vries were more and more refined and, moreover, other mechanisms explaining the absence of layer shrinkage suggested [3,4]. Nevertheless, the hollow cone has been a widely accepted model in the literature and is repeatedly referred to in discussing de Vries behavior or even taken as a basis for theories describing it. However, even after 20 years of extensive investigations, the experimental confirmation of this ad-hoc model is still inconclusive. While optical and electro-optic studies of many de Vries smectics can be readily explained with the hollow cone model, X-ray diffraction studies as well as many polarized Raman spectroscopy studies support a broad sugarloaf ODF.

We review and summarize X-ray, electro-optic and polarized Raman spectroscopy results on the orientational distribution functions in the SmA phases of de Vries-type materials and discuss how seemingly contradicting findings regarding the shape of the ODF can both be true at the same time. Optical properties are governed by the order of the aromatic core, whereas X-ray diffraction essentially probes the order of principal molecular axes. In molecules where the core is far from collinear with the long molecular axis, the cores may exhibit a volcano ODF while the long molecular axes exhibit a sugarloaf ODF.

\section{What determines the smectic layer spacing?}

In the hypothetical case of a SmA phase of rod-like and non-interdigitating molecules with perfect orientational order (Fig. 4a) the smectic layer spacing $d_{A}$ is simply set by the effective molecular length $L_{\text {eff: }}$

$$
d_{A}=L_{e f f}
$$

where $L_{\text {eff }}$ is the thermal average of the molecular length. Note that in the case of partial SmA bi-layer structures, which are quite common in de Vries smectics, $L_{\text {eff }}$ exceeds the molecular length of a single mesogen, depending on the degree of interdigitation.

If orientational fluctuations and thus the imperfect orientational order present in all SmA 

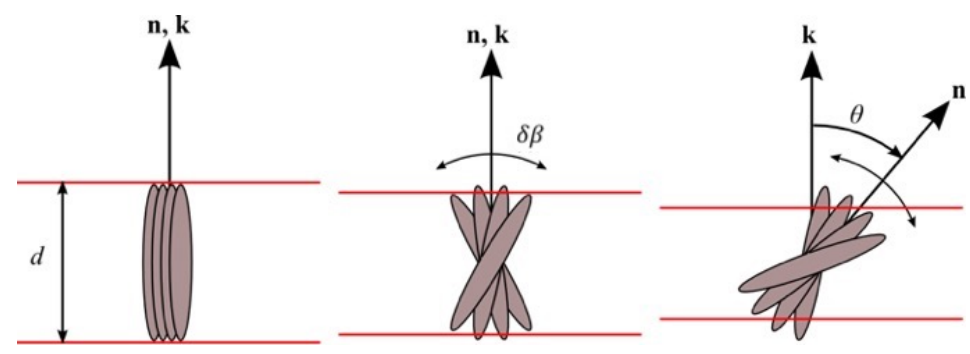

Figure 4. Factors influencing the smectic layer spacing $d$. (a) In SmA with perfect orientational order, $d$ is set by the effective molecular length; (b) in SmA with non-perfect orientational order, $d$ is reduced by molecular orientational fluctuations in $\beta$; (c) the director tilt $\theta$ in SmC further reduces $d$.

phases [3] is taken into account, the layer spacing is reduced to:

$$
d_{A}=L_{e f f}<\cos \beta_{i}>,
$$

where $\beta_{i}$ denote the molecular tilt angles of individual molecules $i$ (Fig. $4 \mathrm{~b}$ ). The ensemble average $\left\langle\cos \beta_{i}\right\rangle$ can be approximated up to second order in $\beta_{i}$ by the orientational order parameter:

$$
S_{2}=\frac{1}{2}\left\langle 3 \cos ^{2} \beta_{i}-1\right\rangle,
$$

leading to [5]:

$$
d_{A} \approx L_{e f f} \frac{S_{2}+2}{3} .
$$

In the $\mathrm{SmC}$ phase the average axis of molecular orientation (the director $\mathbf{n}$ ) is tilted by the director tilt angle $\theta$ (Fig. 4c) and the layer spacing in the $\mathrm{SmC}$ phase becomes $[6,7]$ :

$$
d_{C} \approx L_{\text {eff }} \frac{S_{2}+2}{3} \cos \theta .
$$

This result leads us to the following conclusions:

- There are three main factors - all of them temperature dependent in general - defining the smectic layer spacing: the effective length of the mesogen, the degree of molecular orientational order, and the director tilt.

- There are two options to counteract the reduction in layer spacing due to an increasing director tilt $\theta$ : An increase in effective molecular length $L_{\text {eff }}$ and/or an increase in the orientational order parameter $S_{2}$.

- It has not escaped our notice that any shape of an orientational distribution function may it be sugarloaf or volcano - could result in de Vries behaviour as long as they lead to the same values of $S_{2}$.

Gorkunov et al. have undertaken detailed calculations of the layer spacing in the $\mathrm{SmC}$ phase using a molecular model which corresponds to a sugarloaf-shaped ODF [8]. It has been shown that both a virtually constant layer spacing and the conventional layer contraction can be reproduced using the same intermolecular interaction potential with different values of the coupling constants. In particular, if one neglects the biaxial order parameter in the SmC phase, the following simple relationship is generally valid:

$$
S_{2}^{\prime}=S_{2} P_{2}(\cos \theta),
$$


where the order parameter $S_{2}^{\prime}$ specifies the ordering of long molecular axes with respect to the layer normal $\mathbf{k}$ while the orientational order parameter $S_{2}$ (see Eq.(3)) specifies the ordering with respect to the director $\mathbf{n}$ in the $\mathrm{SmC}$ phase (which is tilted by the angle $\theta$ with respect to $\mathbf{k}$ ). In the case of constant layer spacing the order parameter $S_{2}^{\prime}$ is also constant according to Eq.(4). This can be achieved if the decrease of $P_{2}(\cos \theta)$ with the increasing tilt angle is compensated by the growth of the nematic order parameter $S_{2}$. As shown in [8], such a compensation is possible in a model with a sugarloaf ODF if $S_{2}$ is not too high.

\section{The shape of orientational distribution functions}

In the general theory of orientational order of rod-like molecules in uniaxial liquid crystals such as $\operatorname{SmA}$ the ODF $f(\beta)$ is expanded in terms of even Legendre polynomials $P_{2 L}(\cos \beta)$ :

$$
f(\beta)=\frac{1}{2}+\frac{5}{2} S_{2} P_{2}(\cos \beta)+\frac{9}{2} S_{4} P_{4}(\cos \beta)+\ldots
$$

The expansion coefficients:

$$
S_{2 L}=\left\langle P_{2 L}(\cos \beta)\right\rangle
$$

are known as the orientational order parameters, the first non-trivial of which are the $S_{2}$ given in Eq. 3 and $S_{4}$ :

$$
S_{4}=\frac{1}{8}\left\langle 35 \cos ^{4} \beta-30 \cos ^{2} \beta+3\right\rangle .
$$

Generally the expansion is ended with $P_{4}(\cos \beta)$ as there is only limited experimental knowledge available about higher order terms.

We now have to address the key question how the sugar-loaf and volcano ODFs in Fig. 3 are distinguished in terms of the order parameters $S_{2}$ and $S_{4}$ ? Since a positive value of $S_{2}$ is required for the thermodynamic stability of the liquid crystal phase [9], the only way to obtain the local minimum of the volcano-shaped ODF at $\beta=0$ is a significant negative value of a higher order parameter $S_{2 L}$ with $L \geq 2$. In other words, the volcano-shaped ODF is distinguished from the regular sugar-loaf ODF by negative values of higher orientational order parameters such as $S_{4}$.

This leads to the important consequence, that measurements of the optical birefringence or linear dichroism can in principle not distinguish between sugarloaf and volcano ODFs. Since these properties are related to second-rank tensors, namely the dielectric tensor, their anisotropy 
depends on $S_{2}$ only [10] and thus they neither probe the sign nor the magnitude of $S_{4}$ or any other higher orientational order parameter.

Order parameters $S_{2}$ and $S_{4}$ in the SmC phase as well as profiles of the layer spacing $d$ have been calculated in Ref. [11] for three different shapes of the ODF: hollow cone, volcano like and sugarloaf. It has been shown that for all realistic values of the molecular model parameters the order parameter $S_{4}$ remains positive although it can be rather small for hollow cone distribution with a shallow minimum at the origin. At the same time all models yield very similar temperature variation of the order parameter $S_{2}$ below the transition point into the SmC phase provided the value of $S_{2}$ at the transition is the same. The best qualitative coincidence with typical experimental data is obtained when the ODF in the SmA phase possesses a relatively low flat maximum at the origin which is also compatible with low values of $S_{2}$.

\section{Experimental findings - $S_{2}, S_{4}$}

With the renewed interest in de Vries-type smectics, the orientational order parameters of these SmA phases were intensively studied by various experimental methods, namely $2 \mathrm{D} \mathrm{X}$ ray diffraction (XRD), polarized Raman spectroscopy (PRS) and infrared dichroism (IRD). 2D XRD studies of well aligned SmA samples enable in principle to obtain the full ODF and thus all order parameters $S_{2}, S_{4}, S_{6}, \ldots$ by a detailed analysis of the azimutal intensity distribution $I(\chi)$ of the diffuse wide-angle scattering originating from the fluid intra-layer correlations of the molecules [12-14]. PRS probes the principal axis of a fourth-rank tensor - the Raman tensor and thus enables measurements of the order parameters $S_{2}$ and $S_{4}$ [15-18]. As already outlined above, measurements of linear dichroism such as IRD are limited to $S_{2}$.

A comprehensive selection of order parameters found with these methods for de Vries-type $\mathrm{SmA}$ (or $\mathrm{SmA}^{*}$ ) phases is presented in Tab. 1. We first of all notice from Tab. 1 that most values of $S_{2}$ are in the range between 0.4 and 0.6 , some of them even below 0.4 . This range of $S_{2}$ is unusually small for SmA phases: McMillan's theory of SmA phases predicts the orientational order parameter to be in the typical range of $S_{2}=0.7 \ldots 0.9$ [19] which was later also confirmed by experimental results, see e.g. [12,13,15,20,21]. In contrast, the de Vries-type SmA phase is signified by an unusually weak orientational order, the corresponding $S_{2}$ values of which are in the typical range of nematic phases $\left(S_{2}=0.4 \ldots 0.7\right.$ according to the Maier-Saupe theory [9] or even below.

This observation led to the far-reaching conclusion that orientational ordering is obviously not the main driving force in the formation of a de Vries-type SmA phase [31]. Instead, the formation of de Vries-type SmA is driven by a strong tendency to smectic layering, i.e. by the nanosegregation of chemically incompatible molecular segments [2]. In this case, the smectic 1D translational order parameter $\sigma$ is the primary order parameter of the (direct!) isotropic to SmA transition while the orientational order parameter $S_{2}$ - as a secondary order parameter can in principle remain small. This consideration does not only explain why there is no example of a de Vries-type material with a nematic phase, it also rationalizes current design strategies of de Vries-type smectics which are all based on the incorporation of nanosegregating molecular segments such as terminal siloxane or silane chains.

In non-de Vries materials which follow the McMillan theory, the orientational order parameter $S_{2}$ is primary while the smectic $1 \mathrm{D}$ translational order parameter $\sigma$ is a secondary order parameter which becomes non zero at sufficiently high orientational order. In this conventional case $S_{2}$ is high and almost saturated in the SmA phase and thus can not further increase to an extent which would be necessary to compensate the layer shrinkage in a transition to SmC. Low values of $S_{2}$ are thus essential for a de Vries-type SmA phase.

We further notice from Tab. 1 that measured values of $S_{4}$ (and $S_{6}$ in [26]) are all positive or close to zero. At least, there is so far no experimental evidence of clear negative values of $S_{4}$ or of any other higher order parameter which however would be required to describe a volcanoshaped ODF (cf. Section 3). In this sense, the experimental values of $S_{2}$ and $S_{4}$ known so far do not support the hollow cone model or the presence of a volcano ODF. Instead, the rather 
Table 1. Compilation of orientational order parameters $S_{2}, S_{4}$ and $S_{6}$ obtained for de Vries-type SmA phases by 2D $\mathrm{X}$-ray diffraction (XRD), infrared dichroism (IRD) and polarized Raman scattering(PRS).

\begin{tabular}{rllccc}
\hline \hline Ref. & Material & Method & $S_{2}$ & $S_{4}$ & $S_{6}$ \\
\hline$[5]$ & 3M & XRD & 0.56 & $>0$ & - \\
\hline$[22]$ & DSiKN65 & IRD & 0.48 & - & - \\
& TSiKN65 & IRD & 0.38 & - & - \\
\hline$[23]$ & TSiKN65 & PRS & $0.25 \ldots 0.30$ & $-0.1 \ldots 0.0$ & - \\
\hline$[24]$ & TSiKN65 & IRD & $0.4 \ldots 0.6$ & - & - \\
\hline$[7]$ & C4 & XRD & $0.7^{\mathrm{a}}$ & - & - \\
& & & $0.4^{\mathrm{b}}$ & - & - \\
& C9 & XRD & $0.6^{\mathrm{a}}$ & - & - \\
& & & $0.4^{\mathrm{b}}$ & - & - \\
\hline$[25]$ & $9 H L$ & XRD & 0.6 & 0.2 & - \\
& & PRS & $0.6 \ldots 0.8$ & $0.1 \ldots 0.5$ & - \\
\hline$[6]$ & 1 (A in [26]) & XRD & 0.45 & $>0$ & - \\
& 2 (B in $[26])$ & XRD & 0.45 & $>0$ & - \\
\hline$[26]$ & A & XRD & 0.5 & 0.15 & 0.05 \\
& B & XRD & 0.5 & 0.15 & 0.05 \\
& C4 & XRD & 0.7 & 0.35 & 0.1 \\
& C9 & XRD & 0.5 & 0.15 & 0.05 \\
\hline$[26]$ & QL13-3 & XRD & $0.43 \ldots 0.47$ & - & - \\
& QL18-3 & XRD & $0.48 \ldots 0.52$ & - & - \\
& QL19-6 & XRD & $0.55 \ldots 0.58$ & - & - \\
\hline$[27]$ & 9HL & NMR & $0.65 \ldots 0.80^{\mathrm{c}}$ & & - \\
\hline$[28]$ & MC 513 & PRS & 0.51 & 0.14 & - \\
\hline$[29]$ & C4 & IRD & $0.3 \ldots 0.4^{\mathrm{a}}$ & - & - \\
& & IRD & $0.4^{\mathrm{b}}$ & - & - \\
- & & IRD & $0.6 \ldots 0.7^{\mathrm{c}}$ & - & - \\
& C9 & IRD & $0.30 \ldots 0.35^{\mathrm{a}}$ & - & - \\
& & IRD & $0.43^{\mathrm{b}}$ & - & - \\
\hline$[30]$ & MSi3MR11 & XRD & $0.45^{\mathrm{c}}$ & - & - \\
\hline \hline & & & & - & - \\
\hline
\end{tabular}

a hydrocarbon segments

b siloxane segments

${ }^{\mathrm{c}}$ mesogenic core 
small but positive values of $S_{2}$ and $S_{4}$ indicate the presence of a broad sugarloaf ODF which can explain the practical absence of layer shrinkage as well (see Section 2) and thus makes the assumption of hollow cone or volcano ODFs unnecessary. This view receives further support from theoretical calculations $[8,11,32]$ and recent simulation results [33].

Even though the experimental values of $S_{2}$ and $S_{4}$ support the assumption of a broad sugarloaf ODF, they do not strictly exclude the existence of volcano ODFs since these might also originate from a negative $S_{6}$ order parameter. Neither the sign nor the magnitude of higher order parameters such as $S_{6}$ has been systematically studied so far, except for the positive $S_{6}$ data reported in [30].

\section{Electro-optics of chiral de Vries smectics - the electroclinic effect}

In chiral smectic $\mathrm{A}\left(\mathrm{SmA}^{*}\right)$ an electric field $\mathbf{E}$ applied along the smectic layers induces a tilt $\theta$ of the director $\mathbf{n}$ in the direction normal to $\mathbf{E}$. This has become known as the electroclinic effect in $\mathrm{SmA}^{*}$ [34]. In $\mathrm{SmA}^{*}$ phases of the de Vries-type the induction of the director tilt is coupled to a substantial increase in birefringence $[2,5,30,35-38]$ which reflects the increase in orientational order associated to the appearance of tilt. Studies of the electroclinic effect thus provide a perfect tool to study the coupling between tilt and orientational order in de Vries-type smectics.

Clark et al. studied the electroclinic effect in two de Vries-type materials and analyzed the results in terms of the hollow cone scenario [37]. In the absence of external electric fields the molecules were considered to be randomly distributed on a cone with the opening angle $2 \beta_{A}$. Under the application of an electric field the molecular dipoles couple to the field and the molecules were assumed to become azimuthally biased towards one side of the cone, resulting in a tilt $\theta$ of the director, and an increase of birefringence $\Delta n$. The exact behaviour of the fieldcontrolled birefringence in this scenario, calculated using the Langevin model for 2D biasing of dipoles [39], is well approximated by [37]:

$$
\frac{\Delta n_{\phi}}{\Delta n_{\max }} \approx P_{2}\left(\cos \beta_{A}\right)+\left(1-P_{2}\left(\cos \beta_{A}\right)\right)\left(\frac{\theta}{\beta_{A}}\right)^{2}
$$

where $\Delta n_{\phi}$ is the birefringence as function of azimuthal biasing of the molecules on the cone, $\Delta n_{\max }$ is the birefringence of the fully switched state, i.e. when all molecules are on the same azimuthal position $\phi$ on the cone, and $P_{2}$ is the second order Legendre polynomial in $\cos \beta_{A}$. Fig. 6 from Ref. [37] shows calculated (using Eq. 10) as well as measured birefringence data for the material studied. It is evident that the strict hollow cone model with a fixed $\beta_{A}$ fails to describe the electroclinic behavior as it predicts a significantly steeper slope in the birefringence vs. induced tilt characteristics than the one observed. But when letting the cone angle increase with director tilt from $\beta_{A} \approx 25^{\circ}$ at $\theta^{2}=0$ to $\beta_{A} \approx 34^{\circ}$ at $\theta^{2} \approx 0.35$ the data could be fairly well represented, cf. Fig. 6 . This apparent field-induced opening of the cone was attributed to the conventional electroclinic effect comprising a tilt of the rod-like molecules in addition to the azimuthal biasing on the cone [38].

Similar investigations were done by other groups as well $[5,36,40]$ and all led to the conclusion that the $\Delta n(\theta)$-coupling is well described by the assumption of Langevin-like ordering of molecules distributed on a hollow cone. This certainly gives strong support to the hollow cone model. But on the other hand, as we pointed out earlier, birefringence does not probe higher order orientational order parameters and thus does not distinguish between sugarloafand volcano-shaped ODFs. It can thus not be excluded that a similar description of experimental $\Delta n(\theta)$ data might also be obtained by models assuming a (broad) sugarloaf ODF with same range of $S_{2}$ as the current hollow-cone based models.

In this context it might be worth to point out that a broad sugarloaf ODF can always be composed by a sum of several volcano ODFs, the opening angles of which decrease from one cone to the next (Fig. 7). In this case, the $\Delta n(\theta)$ coupling should be a superposition of Langevin-type 


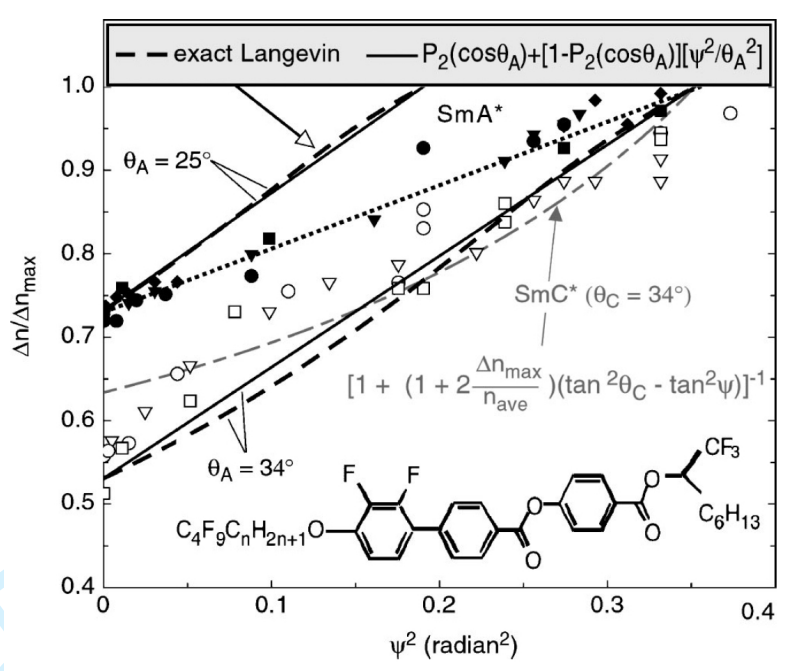

Figure 6. Electroclinic effect in the de Vries material C4, from ref.[37]. The hollow cone model cannot describe the observed birefringence behavior. At $\left(\theta^{2}=\right) \psi^{2}=0$ the birefringence matches a hollow cone with $\left(\beta_{A}=\right) \theta_{A}=25^{\circ}$ while at $\left(\theta^{2}=\right) \psi^{2}=0.33$ the birefringence matches a hollow cone with $\left(\beta_{A}=\right) \theta_{A}=34^{\circ}$. Filled symbols refer to data from the $\mathrm{SmA}^{*}$ phase.

ordering of molecules distributed on hollow cones with different opening angles.

\section{The role of molecular shape}

The positive $S_{4}$ values determined by X-ray diffraction and polarized Raman spectroscopy suggest a sugarloaf ODF for de Vries smectics. However, optics, electrooptic, and linear dichroism measurements provide at least indirect support of a volcano ODF. The reason is that in order to reach the very low values of $S_{2} \approx 0.4$ determined from birefringence and linear dichroism, cf. Tab. 1 a sugarloaf ODF would necessarily have to be unusually broad (like in a nematic) while a volcano ODF can be much narrower and still produce the same small birefringence and, hence, the same low values of $S_{2}$.

We suggest that these seemingly contradicting findings - the sugarloaf ODF from X-ray diffraction and Raman spectroscopy and the volcano ODF from birefringence and dichroism - could in fact be true at the same time. The reason is that the refractive indices and thus the birefringence $\Delta n$ are related to the anisotropic polarizability of the molecules while X-ray scattering basically probes the principal axis of the electron density which is given by the axis of molecular gyration (for a detailed discussion see [41]). This means that birefringence measurements probe the orientational order of the aromatic, highly polarizable, molecular cores, whereas X-ray diffraction probes the orientational order of the long axis of the molecule. Obviously, when we consider molecules where the aromatic core is significantly tilted with respect to the principal molecular axis, the cores may indeed exhibit a volcano ODF while the molecular axes simultaneously exhibit a sugarloaf ODF (see Fig. 8).

To further illustrate the relevance of this picture we focus on the electroclinic effect in a $\mathrm{SmA}^{*}$ phase of strongly Z-shaped molecules [42]. Let us first consider the extreme case illustrated in Fig. 9, where Z-shaped molecules, with the core tilted the angle $\omega$ with respect to the molecular axis a, are perfectly ordered along $\mathbf{k}$, i.e. $S_{\mathbf{a}}=1$, where $S_{\mathbf{a}}$ is the $S_{2}$ orientational order parameter of the molecular axes $\mathbf{a}$. The transverse dipole $\mathbf{p}$ is assumed to be always normal to $\mathbf{k}$ and $\mathbf{a}$. As the molecules are free to rotate about $\mathbf{a}$, the distribution of the molecular cores is mathematically equivalent to the hollow cone model with the opening angle $2 \omega$, and a hollowcone type electroclinic response according to Eq. 10 can in principle occur as a result from pure rotational biasing about $\mathbf{k}$, i.e., with no tilt of the molecular axes. 


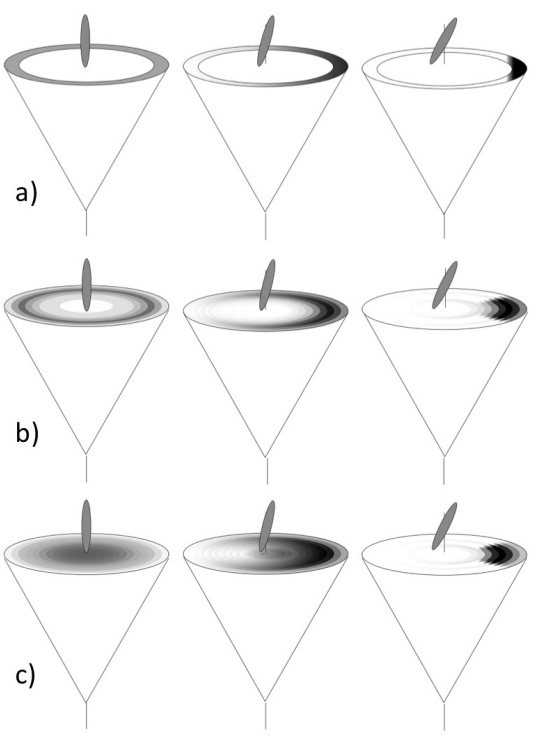

Figure 7. Illustration of the electroclinic effect in an extreme hollow cone scenario (a), in a diffuse cone scenario (volcano ODF) (b) and a sugarloaf ODF (c). Distributions in (b) and (c) can both be constructed by a set of distributions in (a) with varying cone angles.
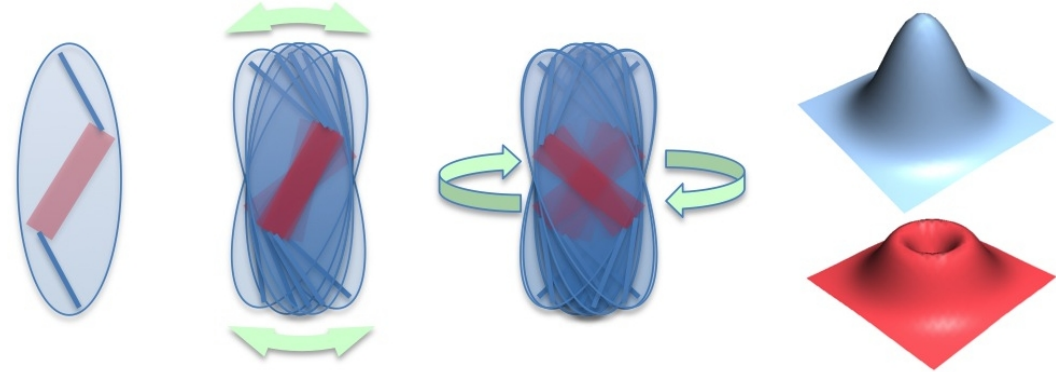

Figure 8. (a) Representation of a molecule (blue ellipsoid) in which the molecular core (red) is significantly tilted away from the principal molecular axis. Tilt fluctuations (b) and azimuthal rotation about the molecular axis (c) gives a sugarloaf ODF of the molecules and a volcano ODF (diffuse cone) of the cores (d).

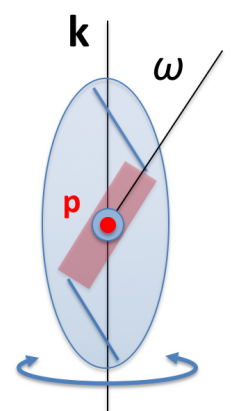

a)

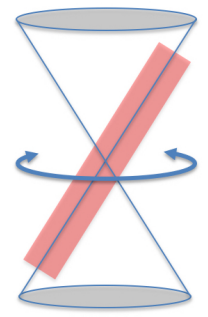

b)

Figure 9. (a) Schematic illustration of a non-tilted, biaxial Z-shaped molecule where the core is tilted the angle $\omega$ with respect to the molecular axis $\mathbf{a}$. With no orientational disorder in the molecular axis, i.e. $S_{\mathbf{a}}=1$ the cores are randomly distributed on a hollow cone with opening angle $2 \omega(\mathrm{b})$. Under an electric field applied normal to $\mathbf{k}$ the molecular dipoles $\mathbf{p}$ are biased along the field direction and the core distribution is tilted and focussed towards one point on the cone (see Fig. 7a). 


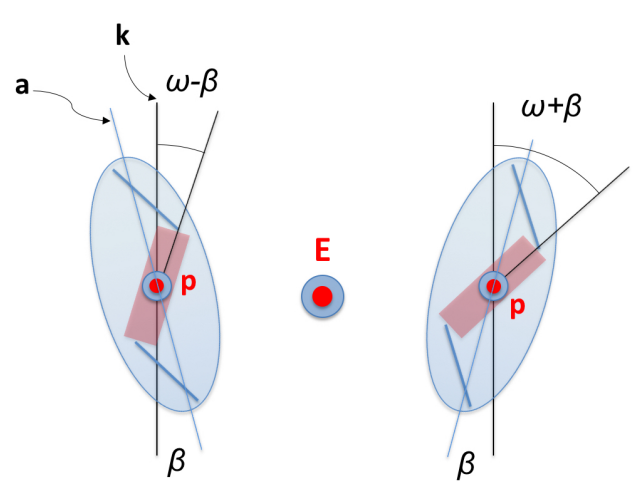

Figure 10. Zigzag biaxial molecules with orientational disorder in molecular axes a about the layer normal k. Under electric field $\mathbf{E}$ the molecular dipoles $\mathbf{p}$ are along $\mathbf{E}$.

Let us now assume a more realistic picture, where the molecular axes a are not perfectly ordered along k, i.e. $S_{\mathrm{a}}<1$. The $S_{2}$ orientational order parameter of the aromatic cores $S_{\text {core }}$ is in turn coupled to $S_{\mathrm{a}}$ through the addition theorem of Legendre polynomials [43]:

$$
S_{\text {core }}=S_{\mathrm{a}} P_{2}(\cos \omega)
$$

For simplicity, let the molecular axes fluctuate between the angles $+\beta$ and $-\beta$ in the plane normal to the applied field $\mathbf{E}$ and let the dipole $\mathbf{p}$ be along $\mathbf{E}$ under electric field. We can then make a rough estimation of $S_{\text {core }}$ determined by the averages $\left\langle\cos ^{2}(\omega+\beta)\right\rangle$ and $\left\langle\cos ^{2}(\omega-\beta)\right\rangle$ in the two cases in Fig. 10 and get:

$$
\cos (2 \omega)\langle\cos 2 \beta\rangle=\frac{4 S_{\mathbf{a}}-1}{3} .
$$

With finite disorder in molecular axes a about the layer normal k, Eq. 10 is then modified according to

$$
\frac{\Delta n}{\Delta n_{\max }} \approx P_{2}\left(\cos \beta_{A}\right)+\frac{4 S_{\mathbf{a}}-1}{3}\left(1-P_{2}\left(\cos \beta_{A}\right)\right)\left(\frac{\theta}{\beta_{A}}\right)^{2}
$$

This means that the slope of birefringence vs. tilt squared should become smaller by a factor $\frac{4 S_{\mathrm{a}}-1}{3}$ when we introduce disorder in the molecular axes, schematically illustrated in Fig. 11a. With $S_{\mathrm{a}}=0.65$, and $\omega=\theta_{A}=25^{\circ}$, we can by using Eq. 13 in fact fairly well match the experimental data for $\mathrm{C} 4$ in Fig. 6, see Fig. 11b. In Fig. 11b is also plotted the calculated birefringence as function of director tilt using Eq. 10 for $\beta_{A}=25^{\circ}$, and $\beta_{A}=34^{\circ}$, from Fig. 6 , as for comparison.

\section{Conclusions}

Even after more than 20 years of investigations, the detailed underlying mechanisms for nonlayer shrinkage behaviour in de Vries smectics are still not clear. The original hollow cone model of de Vries as well as the more general model of a volcano-shaped ODF explain the absence of layer shrinkage and the coupling between birefringence and director tilt in such a simple, intuitive and generic way that they are still widely used. On the other hand, experimental studies of orientational order parameters in de Vries-type SmA phases clearly do not support the existence of a hollow cone or a volcano ODF. These results instead suggest the presence of broad sugarloaf ODFs which exhibit the same low values of $S_{2}$ as the volcano ODFs and which 


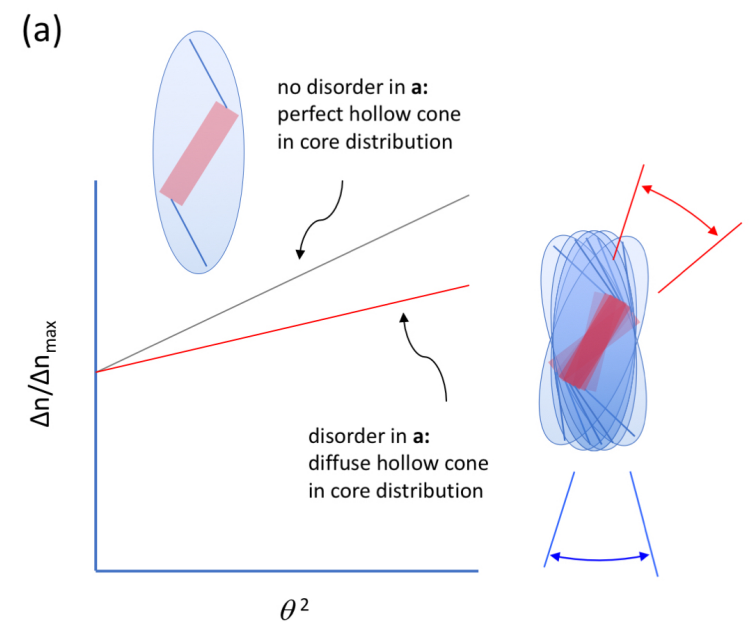

(b)

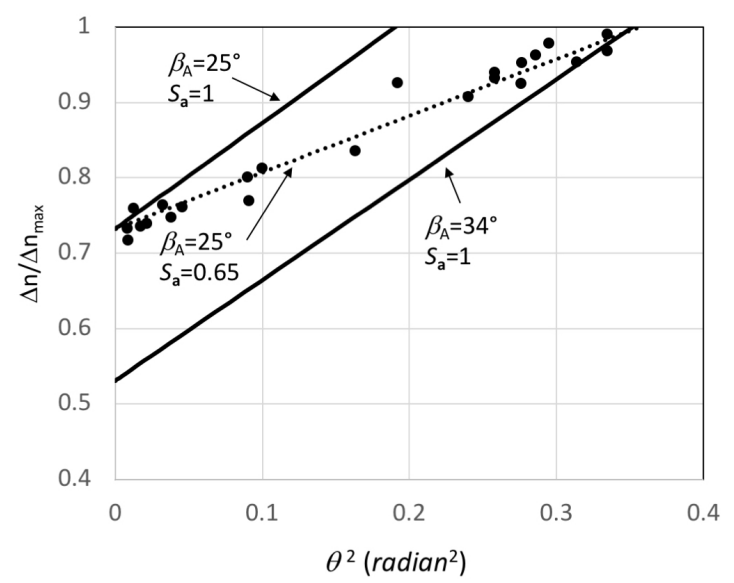

Figure 11. (a) The effect of introducing disorder in molecular axis a, i.e., $S_{\mathrm{a}}<1$ is that the slope of $\frac{\Delta n}{\Delta n_{\max }}$ vs. $\theta^{2}$ is reduced with the factor $\frac{4 S_{\mathrm{a}}-1}{3}$. (b) Calculated birefringence vs. director tilt in the case of a perfect hollow cone scenario with $\beta_{A}=25^{\circ}$, and $\beta_{A}=34^{\circ}$ for the data in Ref. [37] and for the case of orientational disorder in the molecular (or cone) axis $\left(S_{\mathrm{a}}=0.65, \omega=\beta_{A}=25^{\circ}\right.$. The latter case matches the observed behavior, the black dots are the SmA* data from Fig. 6 . 


\section{Acknowledgements}

We thank Noel A. Clark, Joseph E. Maclennan, Mathew. A. Glaser, David M. Walba and Robert P. Lemieux for our fruitful collaboration in the Materials World Network and many stimulating discussions on de Vries smectics. This work was supported by the Deutsche Forschungsgemeinschaft (DFG) and the US National Science Foundation (NSF) in the framework of the NSF/DFG 'Materials World Network' programme (DFG Gi 243/5-1 and DFG Gi 243/6-1) as well as by the Swedish Research Council (VR) Grant No. 621-2009-3621.

\section{References}

[1] de Vries A. Experimental Evidence Concerning Two Different Kinds of Smectic C to Smectic A Transitions. Molecular Crystals And Liquid Crystals. 1977;41(2):27-31.

[2] Lagerwall JPF, Giesselmann F. Current topics in smectic liquid crystal research. ChemPhysChem. 2006 Jan; $7(1): 20-45$.

[3] de Vries A, Ekachai A, Spielberg N. Why the Molecules are Tilted in all Smectic A Phases, and How the Layer Thickness Can be Used to Measure Orientational Disorder*. Molecular Crystals And Liquid Crystals. 1979;49(5):143-152.

[4] de Vries A. The Implications of the Diffuse-Cone Model for Smectic A and C Phases and A-C Phase Transitions. Molecular Crystals And Liquid Crystals. 1979;49(6):179-185.

[5] Lagerwall J, Giesselmann F, Radcliffe M. Optical and x-ray evidence of the "de Vries" Sm-A*$\mathrm{Sm}-\mathrm{C}^{*}$ transition in a non-layer-shrinkage ferroelectric liquid crystal with very weak interlayer tilt correlation. Physical Review E. 2002;66(3):031703.

[6] Nonnenmacher D, Jagiella S, Song Q, et al. Orientational Fluctuations Near the SmecticA to SmecticC Phase Transition in Two "deVries"-Type Liquid Crystals. ChemPhysChem. 2013 Jul; 14(13):2990-2995.

[7] Yoon H, Agra-Kooijman DM, Ayub K, et al. Direct Observation of Diffuse Cone Behavior in de Vries Smectic-A and -C Phases of Organosiloxane Mesogens. Physical Review Letters. 2011;106(8):087801.

[8] Gorkunov MV, Osipov MA, Lagerwall JPF, et al. Order-disorder molecular model of the smectic-Asmectic-C phase transition in materials with conventional and anomalously weak layer contraction. Physical Review E. 2007;76(5):051706.

[9] Maier W, Saupe A. Eine einfache molekulare Theorie des nematischen kristallinflüssigen Zustandes. Zeitschrift fuer Naturforschung A. 1958;13(7):564-566.

[10] Vertogen G, de Jeu WH. Thermotropic liquid crystals, fundamentals. Berlin: Springer-Verlag; 1988. Chapter 9; p. $167 \mathrm{ff}$.

[11] Osipov M, Pajak G. Molecular theory of the tilting transition in smectic liquid crystals with 
weak layer contraction and diffused cone orientational distribution. Physical Review E. 2012 Feb; 85(2):021701.

[12] Leadbetter AJ, Norris EK. Distribution functions in three liquid crystals from X-ray diffraction measurements. Molecular Physics. 1979;38(3):669-686.

[13] Davidson P, Petermann D, Levelut A. The measurement of the nematic order-parameter by X-Ray scattering reconsidered. Journal De Physique Ii. 1995;5(1):113-131.

[14] Sims MT, Abbott LC, Richardson RM, et al. Considerations in the determination of orientational order parameters from X-ray scattering experiments. Liquid Crystals. 2018;371:1-14.

[15] Jen S, Clark NA, Pershan PS, et al. Polarized Raman-Scattering Studies of Orientational Order in Uniaxial Liquid-Crystalline Phases. Journal of Chemical Physics. 1977;66(10):4635-4661.

[16] Jones W, Thomas D, Thomas D, et al. On the determination of order parameters for homogeneous and twisted nematic liquid crystals from Raman spectroscopy. Journal Of Molecular Structure. 2004;708(1-3):145-163.

[17] Southern CD, Gleeson HF. Using the full Raman depolarisation in the determination of the order parameters in liquid crystal systems. European Physical Journal E. 2007 Oct;24(2):119-127.

[18] Sanchez-Castillo A, Osipov MA, Giesselmann F. Orientational order parameters in liquid crystals: A comparative study of x-ray diffraction and polarized Raman spectroscopy results. Physical Review E. 2010;81(2):021707.

[19] McMillan WL. Simple Molecular Model for the Smectic $A$ Phase of Liquid Crystals. Physical Review A. 1971 Sep;4(3):1238-1246.

[20] Haase W, Fan ZX, Müller HJ. Order parameter and packing studies in nematic and smectic A phases by x-ray diffraction. The Journal of Chemical Physics. 1988;89(5):3317-3322.

[21] Bräuniger T, Fung BM. Relation between the orientational ordering and the tricritical behavior for smectic-A to smectic-C phase transition. Journal of Chemical Physics. 1995 May;102(19):7714-7720.

[22] Collings PJ, Ratna BR, Shashidhar R. Order parameter measurements of dichroic dyes dissolved in smectic liquid crystals that tilt without layer contraction. Physical review E, Statistical physics, plasmas, fluids, and related interdisciplinary topics. 2003 Feb;67(2):021705-8.

[23] Hayashi N, Kato T, Fukuda A, et al. Evidence for de Vries structure in a smectic- A liquid crystal observed by polarized Raman scattering . Physical Review E. 2005 Apr;71(4):041705-8.

[24] Hayashi N, Kocot A, Linehan MJ, et al. Experimental demonstration, using polarized Raman and infrared spectroscopy, that both conventional and de Vries smectic-A phases may exist in smectic liquid crystals with a first-order A-C* transition. Physical Review E. 2006 Nov;74(5 Pt 1):051706.

[25] Sanchez-Castillo A, Osipov MA, Jagiella S, et al. Orientational order parameters of a de Vries-type ferroelectric liquid crystal obtained by polarized Raman spectroscopy and x-ray diffraction. Physical Review E. 2012;85(6):061703.

[26] Agra-Kooijman DM, Yoon H, Dey S, et al. Origin of weak layer contraction in de Vries smectic liquid crystals. Physical Review E. 2014 Mar;89(3):C3-6.

[27] Domenici V, Lelli M, Cifelli M, et al. Conformational Properties and Orientational Order of a de Vries Liquid Crystal Investigated through NMR Spectroscopy. ChemPhysChem. 2014 Jan; 15(7):1485-1495.

[28] Yamada Y, Fukuda A, Vij JK, et al. Renewed focus on the small temperature change of smectic layer spacing in ferroelectric and antiferroelectric LCs. Liquid Crystals. 2015;42(5-6):864-876.

[29] Merkel K, Kocot A, Vij JK, et al. Anomalous temperature dependence of layer spacing of de Vries liquid crystals: Compensation model. Applied Physics Letters. 2016 Jun;108(24):243301-6.

[30] Sreenilayam SP, Rodriguez-Lojo D, Panov VP, et al. Design and investigation of de Vries liquid crystals based on 5-phenyl-pyrimidine and (R,R)-2,3-epoxyhexoxy backbone. Physical Review E. 2017 Oct;96(4-1):042701.

[31] Lagerwall ST, Rudquist P, Giesselmann F. The Orientational Order in So-Called de Vries Materials. Molecular Crystals And Liquid Crystals. 2009;510(1):148-157.

[32] Gorkunov MV, Giesselmann F, Lagerwall JPF, et al. Molecular model for de Vries type smecticA -smectic- C phase transition in liquid crystals . Physical Review E. 2007 Jun;75(6):060701-4.

[33] Jenz F, Osipov MA, Jagiella S, et al. Orientational distribution functions and order parameters in "de Vries"-type smectics: A simulation study. Journal of Chemical Physics. 2016 Oct;145(13):134901-6.

[34] Garoff S, Meyer RB. Electroclinic Effect at the $A-C$ Phase Change in a Chiral Smectic Liquid Crystal. Physical Review Letters. 1977 Apr;38(15):848-851.

[35] Spector M, Heiney P, Naciri J, et al. Electroclinic liquid crystals with large induced tilt angle and small layer contraction. Physical Review E. 2000;61(2):1579-1584.

[36] Selinger JV, Collings PJ, Shashidhar R. Field-dependent tilt and birefringence of electroclinic liquid 
crystals: Theory and experiment. Physical Review E. 2001 Nov;64(6):061705-9.

[37] Clark NA, Bellini T, Shao R, et al. Electro-optic characteristics of de Vries tilted smectic liquid crystals: Analog behavior in the smectic $A^{*}$ and smectic $\mathrm{C}^{*}$ phases. Applied Physics Letters. 2002; 80(22):4097-4099.

[38] Shen Y, Wang L, Shao R, et al. Generalized Langevin-Debye model of the field dependence of tilt, birefringence, and polarization current near the de Vries smectic-A* to smectic- $\mathrm{C}^{*}$ liquid crystal phase transition. Physical Review E. 2013;88(6).

[39] Hayashi N, Kato T, Aoki T, et al. Probable Langevin-like director reorientation in an interfaceinduced disordered $\mathrm{SmC}^{*}$-like state of liquid crystals characterized by frustration between ferroand antiferroelectricity. Physical Review Letters. 2001 Jul;87(1):015701.

[40] Sreenilayam SP, Agra-Kooijman DM, Panov VP, et al. Phase behavior and characterization of heptamethyltrisiloxane-based de Vries smectic liquid crystal by electro-optics, $\mathrm{x}$ rays, and dielectric spectroscopy. Physical Review E. 2017;95(3).

[41] Sims MT, Abbott LC, Cowling SJ, et al. Principal molecular axis and transition dipole moment orientations in liquid crystal systems: an assessment based on studies of guest anthraquinone dyes in a nematic host. Physical Chemistry Chemical Physics. 2016 Dec;19:813-827.

[42] Xu J, Selinger RLB, Selinger JV, et al. Monte Carlo simulation of smectic liquid crystals and the electroclinic effect: The role of molecular shape. Physical Review E. 1999 Nov;60(5):5584-5590.

[43] Lafrance CP, Nabet A, Prud'homme RE, et al. On the relationship between the order parameter $\left\langle p_{2}(\cos \theta)\right\rangle$ and the shape of orientation distributions. Canadian Journal of Chemistry. 1995;73:14971505.

[44] Frank FC. What is the director of a liquid crystal? In: Chandrasekhar S, editor. Liquid Crystals - Proceedings of an International Conference held at the Raman Research Institute, Bangalore, December 3 - 8, 1979; London. Heyden; 1980. p. 1-6. 